\title{
Influence of Turbulent Scalar Mixing Physics on Premixed Flame Propagation
}

\author{
H. Kolla ${ }^{1}$ and N. Swaminathan ${ }^{2}$ \\ ${ }^{1}$ Combustion Research Facility, Sandia National Laboratories, Livermore, CA 94557-0969, USA \\ ${ }^{2}$ Department of Engineering, Cambridge University, Cambridge CB2 1PZ, UK
}

Correspondence should be addressed to N. Swaminathan, ns341@cam.ac.uk

Received 27 January 2011; Revised 26 May 2011; Accepted 20 June 2011

Academic Editor: Andrei N. Lipatnikov

Copyright ( $2011 \mathrm{H}$. Kolla and N. Swaminathan. This is an open access article distributed under the Creative Commons Attribution License, which permits unrestricted use, distribution, and reproduction in any medium, provided the original work is properly cited.

\begin{abstract}
The influence of reactive scalar mixing physics on turbulent premixed flame propagation is studied, within the framework of turbulent flame speed modelling, by comparing predictive ability of two algebraic flame speed models: one that includes all relevant physics and the other ignoring dilatation effects on reactive scalar mixing. This study is an extension of a previous work analysing and validating the former model. The latter is obtained by neglecting modelling terms that include dilatation effects: a direct effect because of density change across the flame front and an indirect effect due to dilatation on turbulence-scalar interaction. An analysis of the limiting behaviour shows that neglecting the indirect effect alters the flame speed scaling considerably when $u^{\prime} / s_{L}^{o}$ is small and the scaling remains unaffected when $u^{\prime} / s_{L}^{o}$ is large. This is evident from comparisons of the two models with experimental data which show that the quantitative difference between the two models is as high as $66 \%$ at $u^{\prime} / s_{L}^{o}=0.3$ but only $4 \%$ at $u^{\prime} / s_{L}^{o}=52.4$. Furthermore, neglecting the direct effect results in a poor prediction of turbulent flame speed for all values of $u^{\prime} / s_{L}^{o}$, and both effects are important for practically relevant values of this velocity ratio.
\end{abstract}

\section{Introduction}

The propagation of a deflagration wave in turbulent medium is a classical problem of turbulent premixed combustion and is of great practical significance in the current climate of energy and environment. Expressions for the mean propagation velocity, referred to as "turbulent flame speed" or "turbulent burning velocity", are often required in practical computational fluid dynamics (CFDs) codes employed in the design of combustion systems. Furthermore, from a theoretical standpoint, turbulent flame speed is a useful analytical tool to assess the general validity of turbulent combustion models [1] for premixed flames. Analytical expressions of turbulent flame speed have been studied in many previous works [2-8]. It is well known from the classical theories [9] that in regimes of practical interest, the turbulent burning rate, and hence turbulent flame speed, is dictated by the rate of turbulent mixing at scales relevant to sustain combustion on the flame surface. A direct relationship between the average burning rate and the small scale mixing rate, known as scalar dissipation rate $\tilde{\epsilon}_{c}$, in large Damköhler number flames can be written as [9]

$$
\overline{\dot{\omega}}_{c}=\frac{2}{2 C_{m}-1} \bar{\rho} \tilde{\epsilon}_{c}
$$

where $C_{m}$ is a model constant of order unity and $\bar{\rho}$ is the average density. The Damköhler number is the ratio of the integral time scale of turbulence to the chemical time scale, $\mathrm{Da}=\left(\Lambda / \delta_{L}^{o}\right) /\left(u^{\prime} / s_{L}^{o}\right)$, where $s_{L}^{o}$ and $\delta_{L}^{o}$ are, respectively, the flame speed and thermal thickness of an unstrained planar laminar flame, and the root-mean-square value of turbulent velocity fluctuations with integral length scale $\Lambda$ is $u^{\prime}$. The subscript $c$ denotes a reaction progress variable, which can be defined using scalar mass fractions or temperature or sensible enthalpy [10]. Here, it is defined using temperature, and it varies from zero in the unburnt mixture to one in the fully burnt mixture. The scalar dissipation rate is defined as $\widetilde{\epsilon}_{c}=\overline{\rho \mathscr{D}\left(\nabla c^{\prime \prime} \cdot \nabla c^{\prime \prime}\right)} / \bar{\rho}$, where 
$\mathscr{D}$ is the diffusivity of progress variable $c$ and $c^{\prime \prime}$ is the Favre fluctuation of $c$. The scalar dissipation rate, which denotes the fine scale mixing rate of hot and cold fluid parcels, embodies the scalar field dynamics, and in turn, influences the rate of chemical reactions, since the mixing of the fluid parcels is what ensures sustained combustion on the flame surface. Equation (1) suggests that the modelling of mean scalar dissipation rate yields a model for the mean burning rate. This approach has been used in $[11,12]$ in conjunction with the Kolmogorov-Petrovskii-Piskunov (KPP) analysis to obtain an expression for the turbulent flame speed.

The KPP theorem [13], which is based on eigenvalue analysis of the Favre averaged transport equation for the progress variable, gives the propagation speed of the leading edge of the flame brush as

$$
S_{T}=2 \sqrt{\frac{v_{t}}{\left(\rho_{u} \mathrm{Sc}_{c}\right)}\left(\frac{\partial \overline{\dot{\omega}_{c}}}{\partial \widetilde{c}}\right)_{\tilde{c} \rightarrow 0},}
$$

where $v_{t}$ is the turbulent kinematic viscosity and $\mathbf{S c}_{c}$ is the turbulent Schmidt number for the scalar $\tilde{c}$. Strictly, this analysis applies for statistically planar flames when the density and turbulence diffusivity are taken to be constant. Furthermore, it requires gradient flux approximation. However, Lipatnikov and Chomiak [1] have shown that the KPP analysis is applicable equally when the density and the diffusivity are not constant. The analysis [11] of statistically nonplanar flames using Taylor's series expansion of Hakberg and Gosman [2] results in an expression similar to (2). In the presence of counter gradient flux, which is known to occur inside the flame brush when the thermochemical effects are stronger than the turbulence effects, Corvellec et al. [14] have noted that the solution to the KPP analysis is limited by the condition at the burnt side $(\tilde{c} \rightarrow 1)$ rather than by the condition at the unburnt side $(\tilde{c} \rightarrow 0)$. However, it is well known that the turbulent scalar flux is gradient at the leading edge of the flame brush where (2) applies. Furthermore, Bray [8] has pointed out that other premixed flame theories including nongradient transport of turbulent scalar flux give flame speed expressions which are equivalent to the KPP expression in (2). The KPP analysis has been used in many past turbulent flame speed studies $[1-4,8,14]$.

Substituting (1) into (2), one obtains

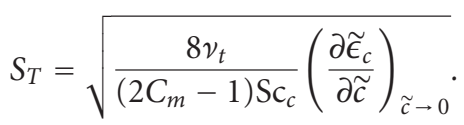

Now, the modelling of turbulent flame speed depends on the scalar dissipation rate modelling. Also, the behaviour of scalar dissipation rate at the leading edge of the flame brush controls the propagation speed, $S_{T}$ as per (3).
Early theories for turbulent flows have taken the scalar dissipation rate to be purely a function of the turbulence parameters. While this is true for turbulent flows with passive scalars, recent studies [15-20] have shown that the scalar dissipation rate is strongly influenced by chemical reactions in premixed flames. The heat release directly affects the local density, which induces alterations in the local dynamics of turbulence, scalar fields, and their interaction and thus, one can envisage a two-way coupling between heat release and the turbulence. This two-way coupling has been investigated in recent studies, and both of these are observed to be leading order [15] effects. A general review of thermal expansion effects is provided by Lipatnikov and Chomiak [21]. Also, algebraic models for the mean scalar dissipation rate and turbulent flame speed that appropriately include these couplings have been proposed recently [12] using the models developed [22] for various terms in the scalar dissipation rate transport equation. These models were validated using turbulent flame speed data covering a wide range of flame configurations and conditions [11].

The aim of the present work is to illustrate the influence of the two-way coupling of heat release effects and the associated physics on turbulent premixed flame propagation. This is done, within the turbulent flame speed framework, by comparing the predictive capability of the flame speed model with and without the terms signifying the physics behind the two-way coupling. In principle, one could also do this within the scalar dissipation rate framework, since the two models have the same terms with the same model parameters [12] as one will find in Section 3. However, the dissipation rate is a very difficult quantity to measure, and experimental data of this quantity are scarce. On the other hand, turbulent flame speed has been widely studied, and there is a wealth of experimental data available for this quantity.

The outline of this paper is as follows. The two-way coupling and the physics associated with the turbulent mixing of a reactive scalar in premixed flames are discussed in Section 2. A brief background on the turbulent flame speed model and the physical significance of various terms is presented in Section 3. The limiting behaviour of the flame speed model which excludes one of the physical effects of dilatation is discussed in Section 4. The analysis of the flame speed expression including the effects of twoway coupling has been reported earlier [11], and thus, it not repeated here. However, relevant results are quoted for convenience. The revised and original flame speed expressions are compared with experimental data in Section 5, and the conclusions of this study are summarised in Section 6.

\section{Physics of Reactive Scalar Mixing}

The physics behind the two-way coupling is explained best using the scalar dissipation rate transport equation, which also helps us to identify the mathematical terms signifying these physical processes. This transport equation has been derived in earlier studies for unity [15] and nonunity Lewis 
number [23] flames. This equation can be written $[15,16$, 22] as

$$
\begin{aligned}
& \bar{\rho} \frac{D \widetilde{\epsilon}_{c}}{D t}-\nabla \cdot\left(\overline{\rho \mathscr{D} \nabla \widetilde{\epsilon}_{c}}\right)=-\underbrace{2 \overline{\rho \mathscr{D}^{2}\left[\nabla\left(\nabla c^{\prime \prime}\right): \nabla\left(\nabla c^{\prime \prime}\right)\right]}}_{D_{2}} \\
& +\underbrace{2 \overline{\rho \epsilon_{c}(\nabla \cdot \mathbf{u})}}_{T_{2}} \\
& -\underbrace{2 \overline{\rho \mathscr{D}\left(\nabla \tilde{c} \cdot \nabla \mathbf{u}^{\prime \prime} \cdot \nabla c^{\prime \prime}\right)}}_{T_{31}} \\
& -\underbrace{2 \overline{\rho \mathscr{D}\left(\nabla c^{\prime \prime} \cdot \nabla \mathbf{u}^{\prime \prime} \cdot \nabla c^{\prime \prime}\right)}}_{T_{32}}
\end{aligned}
$$

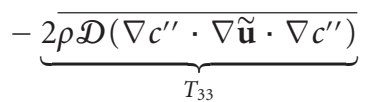

$$
\begin{aligned}
& +\underbrace{2 \overline{\mathcal{D ( \nabla c ^ { \prime \prime } \cdot \nabla \dot { \omega } _ { c } ^ { \prime \prime } )}}}_{T_{4}}
\end{aligned}
$$

for unity Lewis number, where $\rho$ is the density, $\mathbf{u}$ is the velocity vector, and $\dot{\omega}_{c}$ is the reaction rate of $c$. The left hand side of the above equation represents the unsteady, convective and diffusive flux of the scalar dissipation rate in a control volume. On the right hand side, the first term denotes the molecular dissipation effects, and the direct influence of density change across the flame front is denoted by $T_{2}$. These two are leading order terms [15]. The indirect influence of density change through the interaction of turbulence and scalar fields is denoted by the three $T_{3}$ terms. Out of these three terms, $T_{32}$ is identified [15] to be a leading order term in premixed flames. Using Eigen decomposition, this term can be written as $T_{32}=2 \overline{\rho \epsilon_{c}\left(e_{\alpha} \cos ^{2} \theta_{1}+e_{\beta} \cos ^{2} \theta_{2}+e_{\gamma} \cos ^{2} \theta_{3}\right)}$, where $e_{\alpha}>e_{\beta}>e_{\gamma}$ are the principal components of the turbulence strain tensor $\nabla \mathbf{u}^{\prime \prime}$ and $\theta_{i}$ is their orientation angle with $\nabla c^{\prime \prime}$. The contribution of chemical reactions is denoted by $T_{4}$, which is also a leading order term [15], and it will prevail even if the reaction is passive (without heat release). This transport equation has been studied in the past $[15,17]$ to develop physical understanding and to develop models $[22,24]$ for its various terms. Details can be found in these studies.

For a passive chemical reaction, $T_{2}$ is zero. Furthermore, in turbulent flows $T_{32}$ is dictated by the alignment of scalar gradient with the principal turbulent strain rates as noted above. The alignment characteristics are opposite when the chemical reaction is passive or active; the scalar gradient aligns preferentially with the most compressive strain when the chemical reactions are passive, and the scalar gradient aligns preferentially with the most extensive strain when there is strong heat release from the reactions. These situations are schematically shown in Figure 1, and this change is because of the fact that the dilatation due to heat release is strong compared to the turbulent strain rate. Evidence for such a behaviour has been found in direct numerical simulation (DNS) studies of statistically planar [16-20, 22], spherically symmetric [25], and Bunsen [26] flames and also experimental bluff body stabilised flames [27]. As a consequence, the isoscalar surfaces are brought together by the compressive strain resulting in an increase of scalar gradient when the reaction is passive. Because of the change in the alignment, the opposite is true for premixed flames with high heat release. In the present study, we are interested in elucidating the effect of this change in the physics on the propagation speed of the flame brush leading edge. As noted earlier, modelling of these physical process has been done in previous studies [22], and these models have been used [12] to obtain a model for the turbulent flame speed. We start from this model for this study.

\section{Turbulent Flame Speed Model}

A model for the scalar dissipation rate, $\widetilde{\epsilon}_{c}$, accounting for the direct and indirect effects of heat release rate is written as [12]

$$
\tilde{\epsilon}_{c}=\frac{1}{\beta^{\prime}}\left[\left(2 K_{c}^{*}-\tau C_{4}\right) \frac{s_{L}^{o}}{\delta_{L}^{o}}+C_{3} \frac{\widetilde{\varepsilon}}{\widetilde{k}}\right] \widetilde{c^{\prime \prime 2}} .
$$

Using this model in (3), the algebraic model for the flame speed, $S_{T}$, presented in [12] is obtained, and it is written as follows:

$$
\begin{aligned}
\left(\frac{s_{T}}{s_{L}^{o}}\right)=\left\{\frac{18 C_{\mu}}{\left(2 C_{m}-1\right) \beta^{\prime}}[\right. & {\left[2 K_{c}^{*}-\tau C_{4}\right]\left(\frac{u^{\prime} \Lambda}{s_{L}^{o} \delta_{L}^{o}}\right) } \\
& \left.\left.+\frac{2 C_{3}}{3}\left(\frac{u^{\prime}}{s_{L}^{o}}\right)^{2}\right]\right\}^{1 / 2}
\end{aligned}
$$

for turbulent premixed flames with large Reynolds, Re, and Damköhler numbers.

The constant $C_{\mu}$ in (6) is the standard $k-\varepsilon$ turbulence modelling constant with a value of 0.09 and the constant $C_{m}$ $=0.7$ for hydrocarbon-air flames [9]. The physical meaning of other constants is as follows. The symbol $\beta^{\prime}=6.7$ represents the contributions from processes related to flame front curvature, and it comes from the modelling of $\left(T_{4}-\right.$ $D_{2}$ ) in (4). The direct contribution of dilatation, $T_{2}$, at leading order is signified through the term containing $K_{c}^{*}$. The numerical value of $K_{c}^{*}$ is $0.85 \tau$ for hydrocarbon-air mixtures and $0.65 \tau$ for hydrogen-air mixtures, where $\tau \equiv$ $\left(T_{\text {ad }}-T_{u}\right) / T_{u}$ is the heat release parameter, where $T_{u}$ and $T_{a d}$ are the unburnt mixture and adiabatic flame temperatures, respectively. The leading order effects of indirect influence of dilatation through the scalar gradient alignments, $T_{32}$, is signified by the term with $C_{4}$ and $C_{3}$ represents the contribution of turbulence. The values of these two constants are, respectively,

$$
C_{3}=\frac{1.5 \sqrt{\mathrm{Ka}}}{1+\sqrt{\mathrm{Ka}}}, \quad C_{4}=\frac{1.1}{(1+\mathrm{Ka})^{0.4}},
$$

where Ka is the Karlovitz number, which is defined as the ratio of chemical to Kolmogorov time scales, and can be evaluated using $\mathrm{Ka}=\left(\mathscr{D} / s_{L}^{0} \eta\right)^{2}$, where $\eta$ is the Kolmogorov length scale. The ratio of the integral turbulent velocity 


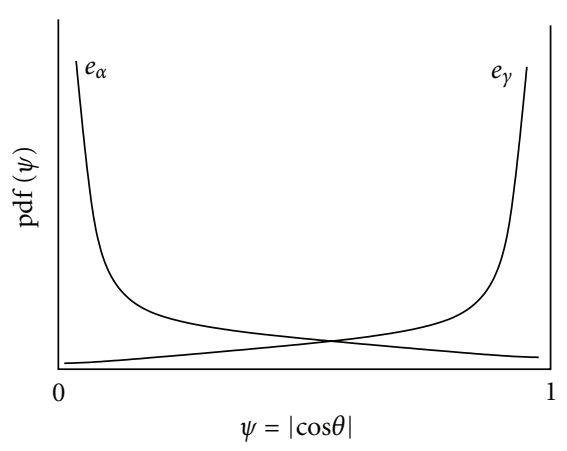

(a)

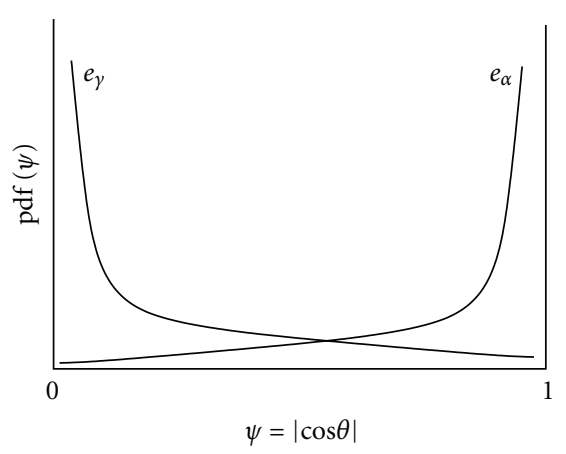

(b)

FIGURE 1: Schematics showing the alignment of scalar gradient with the two principal components of turbulent strain rate tensor $\nabla \mathbf{u}$ in (a) nonreacting turbulence and (b) turbulence with premixed combustion. The most extensive and compressive principal strain rates are noted respectively as $e_{\alpha}$ and $e_{\gamma}$.

time scale to scalar time scale is denoted by $C_{3}$, and its dependence on $\mathrm{Ka}$ is introduced to capture the variation in the value of the time scale ratio [12]. The Ka dependence for $C_{4}$ is introduced in [24] to recover the classical alignment behaviour of the scalar gradient with the principal turbulent strain rates when Ka becomes large.

It is evident that the terms pertaining to the two-way coupling are $K_{c}^{*}$ and $C_{4}$, and both are consequences of dilatation because of chemical heat release. It is worth pointing out that these terms are relevant for compressible fluids and may not be important for the case of, say, chemical reactions occurring in turbulent liquids. The limiting behaviour of (6) without these terms will be studied in the next section.

\section{Influence of Dilatation Effects on Limiting Behaviour}

The case of passive chemical reaction is trivial for this study and has been discussed already in [11]. Since there is no heat release, both $K_{c}^{*}$ and $C_{4}$ are zero for this scenario. Hence, (6) reduces to the classical form [1] $\left(S_{T} / s_{L}^{o}\right) \simeq C\left(u^{\prime} / s_{L}^{o}\right)$ with the constant $C$ having a dependence on the length scale ratio $\Lambda / \delta_{L}^{o}$ (the Karlovitz number here, to be precise). If one includes the molecular viscosity of the fluid in the KPP analysis, then $\left(S_{T} / s_{L}^{o}\right) \simeq 1+C\left(u^{\prime} / s_{L}^{o}\right)$, as has been noted in earlier studies $[11,28]$. This classical expression, without Ka dependence, has been suggested by many turbulent premixed flame theories and is not particularly interesting for the present study.

If one were to retain only the direct part of the two-way coupling, then $K_{c}^{*}$ is nonzero and $C_{4}=0$. This physically implies that the leading order contribution of dilatation due to density change across the flame front is important, but the induced alterations in the dynamics of turbulence and scalar mixing to be ignored. This situation is an improvement from the classical cases noted above, which assume that the alignment characteristics of reactive scalar gradients are the same as those of passive scalars. Now, from (6), one obtains

$$
\left(\frac{S_{T}}{s_{L}^{o}}\right)=\left\{\frac{18 C_{\mu}}{\left(2 C_{m}-1\right) \beta^{\prime}}\left[2 K_{c}^{*}\left(\frac{u^{\prime} \Lambda}{s_{L}^{o} \delta_{L}^{o}}\right)+\frac{2 C_{3}}{3}\left(\frac{u^{\prime}}{s_{L}^{o}}\right)^{2}\right]\right\}^{1 / 2} .
$$

It is worth noting that this feature would appear in a flame speed expression that could be obtained using the mean scalar dissipation rate, $\tilde{\epsilon}_{c}$, model proposed in the earlier work of Swaminathan and Bray [15] in (3).

To study the limiting behaviour of (8) in the limits of low and high $u^{\prime} / s_{L}^{o}$, following [11], we assume $\Lambda / \delta_{L}^{o}$ to be constant and recast $(8)$ as $\left(S_{T} / s_{L}^{o}\right) \sim \sqrt{(A+B)\left(u^{\prime} / s_{L}^{o}\right)^{2}}$, where $A=2 K_{c}^{*} \mathrm{Da}$ and $B=2 C_{3} / 3$.

(i) In the limit of small $u^{\prime} / s_{L}^{o}, \mathrm{Ka}$ is small, and hence, $C_{3} \sim \sqrt{\mathrm{Ka}}$. On the other hand, since $\mathrm{Da}>1, A \sim$ $\mathcal{O}(\tau) \mathrm{Da}$. The value of $\tau$ for most fuels lies typically in the range 5 to 10 , and hence, one would expect $A \gg B$, which yields the scaling

$$
\frac{S_{T}}{s_{L}^{o}} \sim \sqrt{\mathcal{O}(\tau) \frac{u^{\prime} \Lambda}{s_{L}^{o} \delta_{L}^{o}}}
$$

suggesting that $S_{T} / s_{L}^{o} \sim\left(u^{\prime} / s_{I}^{o}\right) \sqrt{\mathrm{Da}}$ or $S_{T} / u^{\prime} \sim \sqrt{\mathrm{Da}}$, which is different from $\mathrm{Da}^{1 / 4}$ discussed in [1]. The scaling in (9) suggests a square root dependence on $\left(u^{\prime} / s_{L}^{o}\right)$ for the normalised turbulent flame speed, $S_{T} / s_{L}^{o}$, and also the contribution of thermochemistry overwhelms the contribution of turbulence for small $u^{\prime} / s_{L}^{o}$ values. On the other hand, the opposite is true for (6) which yields a linear scaling for $S_{T} / s_{L}^{o}$ with $u^{\prime} / s_{L}^{o}$. This is because the contribution of direct effect of dilatation, signified by $K_{c}^{*}$ term, is balanced by the contribution of $C_{4}$ making $A$ to be of order zero for (6) as has been noted in [11]. The linear scaling is consistent with the scaling arguments proposed by Damköhler [29]. 


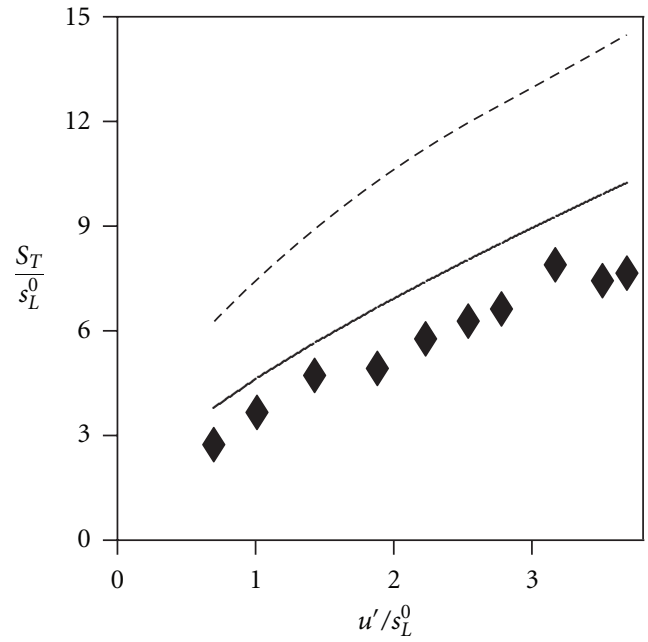

(a)

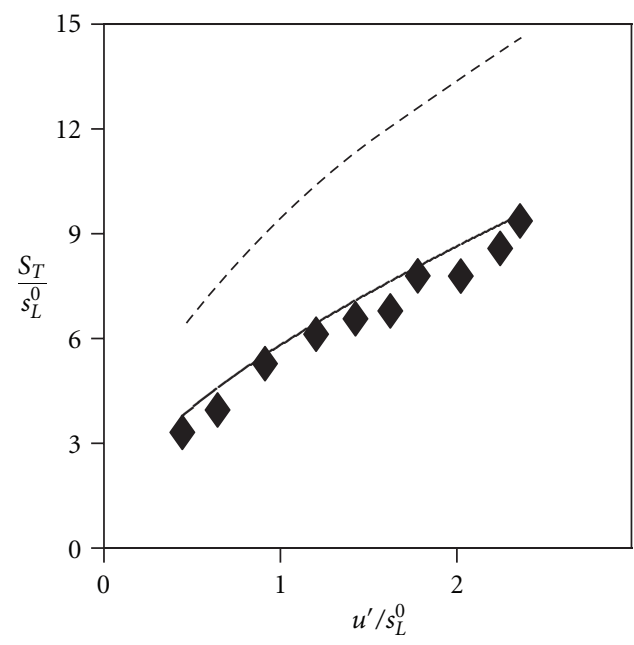

(c)

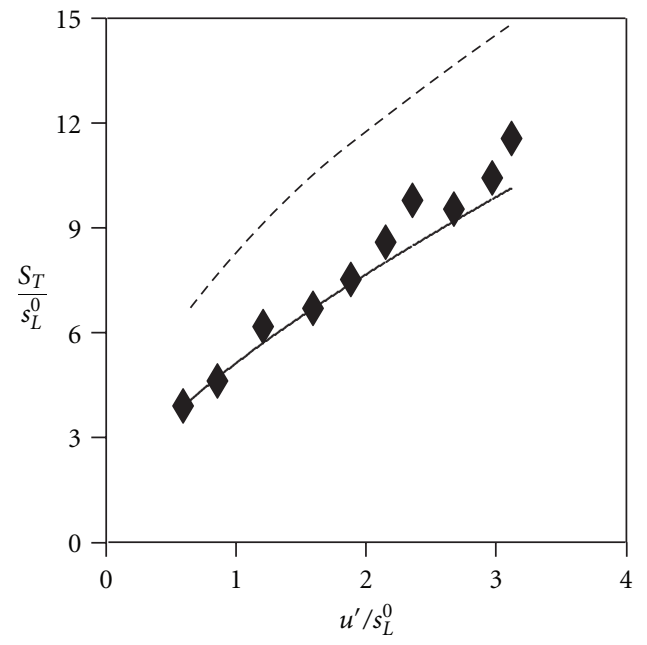

(b)

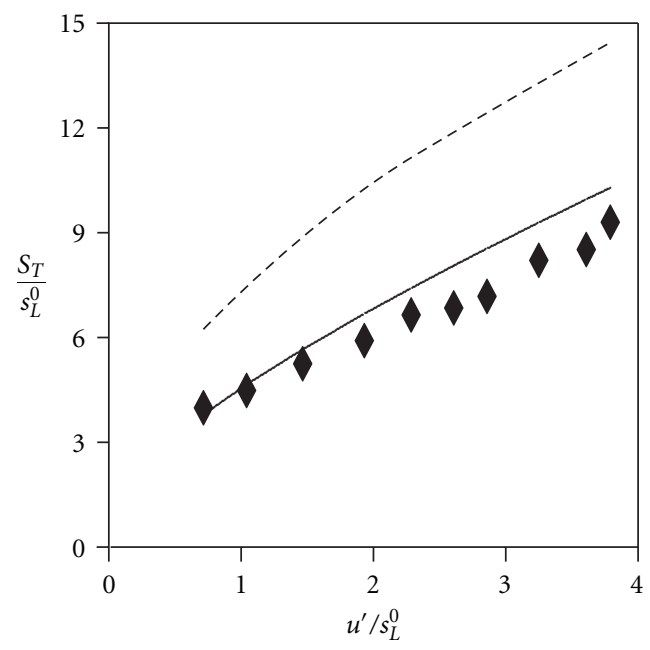

(d)

Figure 2: The predictions of turbulent flame speed expressions, (6) (solid line) and (8) (dashed line), are compared to the experimental data of [31] for various equivalence ratios: (a) $\phi=0.8$; (b) 1.0 ; (c) 1.2 ; (d) 1.4 .

(ii) In the limit of large $u^{\prime} / s_{L}^{o}, \mathrm{Ka}$ is large and $C_{3} \approx 1.5$. Hence, $A \sim \mathcal{O}(\tau) \mathrm{Da}$ and $B \sim \mathcal{O}(1)$ which gives the scaling

$$
\frac{S_{T}}{s_{L}^{o}} \sim \sqrt{\mathcal{O}(\tau) \frac{u^{\prime} \Lambda}{s_{L}^{o} \delta_{L}^{o}}+\mathcal{O}(1)\left(\frac{u^{\prime}}{s_{L}^{o}}\right)^{2}},
$$

for both (6) and (8), since $C_{4} \simeq 0$ for large Ka. The above scaling can also be written as $S_{T} / u^{\prime} \sim$ $\sqrt{1+\mathcal{O}(\tau) \mathrm{Da}}$.

It is interesting to note that neglecting $C_{4}$-related term affects the scaling of turbulent flame speed in the limit of small $u^{\prime} / s_{L}^{o}$ but not in the limit of large $u^{\prime} / s_{L}^{o}$. This is not surprising, since $C_{3}$ and $C_{4}$ represent the competing effects of turbulent straining and thermochemistry respectively on the interaction between turbulence and scalar fields [16, 22]. When $u^{\prime} / s_{L}^{o}$ is very large, the turbulence is expected to overwhelm the thermochemical process, and the model parameters reflect this behaviour. Hence, neglecting $C_{4}$ makes no difference in the large $u^{\prime} / s_{L}^{o}$ limit. (It is also important to note that these scaling does not say how large is large.) This does not imply that the direct effect of dilatation through $K_{c}^{*}$ is also negligible, and its influence appears as the first term in (10). Also, the above two scaling expressions for the turbulent flame speed suggests a qualitative difference when compared to the corresponding classical scaling relations. This is somewhat different from the observation in [30], suggesting only a quantitative influence of density change for the propagation speed of premixed flames in large-scale and low-intensity turbulence.

Finally, it is worth commenting on why we have not considered the case where $K_{c}^{*}$ is neglected but $C_{4}$ is retained. Such a model is not physically correct, since it ignores the leading order contribution of dilatation, and it would not satisfy the realisability condition for the mean scalar 


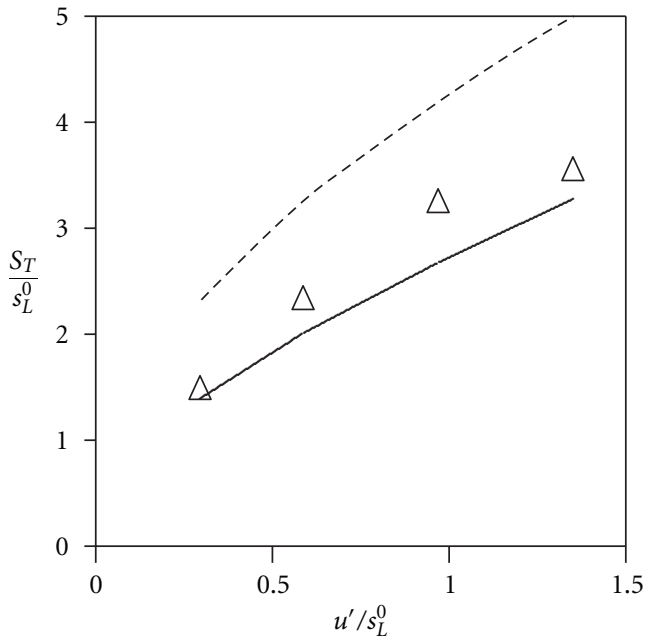

(a)

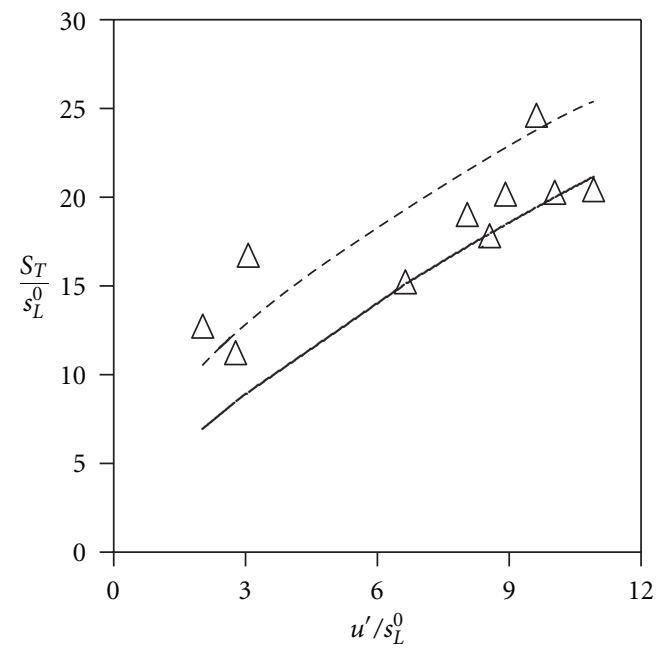

(c)

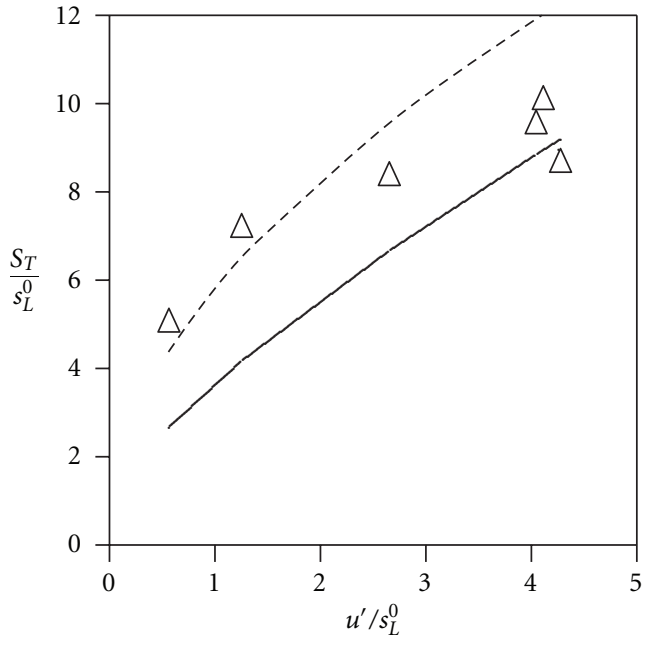

(b)

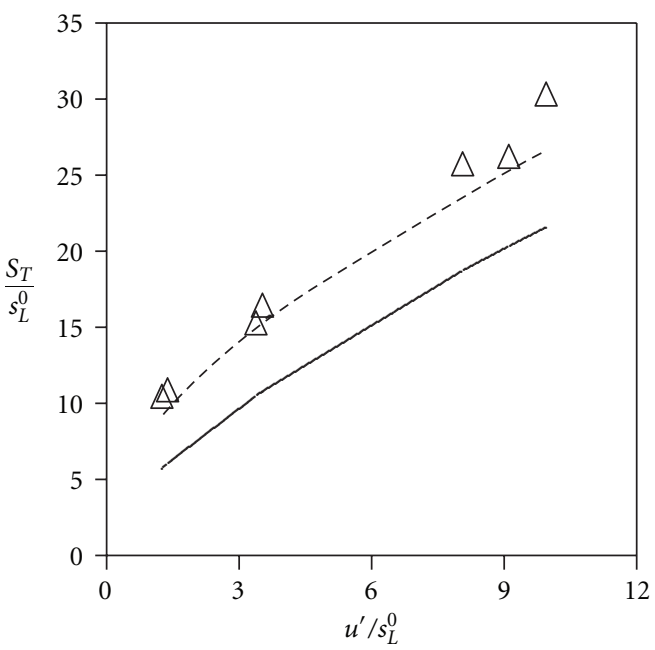

(d)

Figure 3: The predictions of the flame speed expressions, (6) (solid line) and (8) (dashed line), are compared to the high pressure Bunsen flame data of [33]: (a) $0.1 \mathrm{MPa}$; (b) $0.5 \mathrm{MPa}$; (c) $2 \mathrm{MPa}$; (d) $3 \mathrm{MPa}$.

dissipation rate discussed in [12]. Physically, setting $K_{c}^{*}=0$ implies that there is no density change across the flame front, and thus, it is meaningless to consider the indirect influence of density change through the dynamics of turbulencescalar interaction. Furthermore, for low values of $\mathrm{Ka}$, such a model might yield negative value of mean scalar dissipation rate which is unphysical. In the next section, the predictive capability of (8) is compared to that of (6) by making comparisons with experimental data.

\section{Comparison to Measurements}

The original flame speed model, (6), was comprehensively validated in [11] with experimental data from a wide range of turbulent flame configurations and conditions spanning all regimes of practical interest. Particularly, due rigour was paid to the definition of turbulent flame speed given by the KPP analysis - the propagation speed of flame brush leading edge- and the experimental data were chosen accordingly. Other definitions of turbulent flame speed are possible and the reader is referred to the review paper by Driscoll [32] or [11] for a discussion on this topic. For comparisons in the present study, we choose the following subset of the experimental data considered in [11]:

(1) the planar flame data from the Taylor-Couette apparatus of Aldredge et al. [31],

(2) the high pressure Bunsen flame data of Kobayashi et al. [33],

(3) the very high turbulence intensity data of Il'yashenko and Talantov $[34,35]$.

This is to emphasis the relative roles of direct and indirect effects of density change on the propagation speed of the flame brush leading edge for wide conditions of turbulent flames. As noted earlier, ignoring the effects of density change 


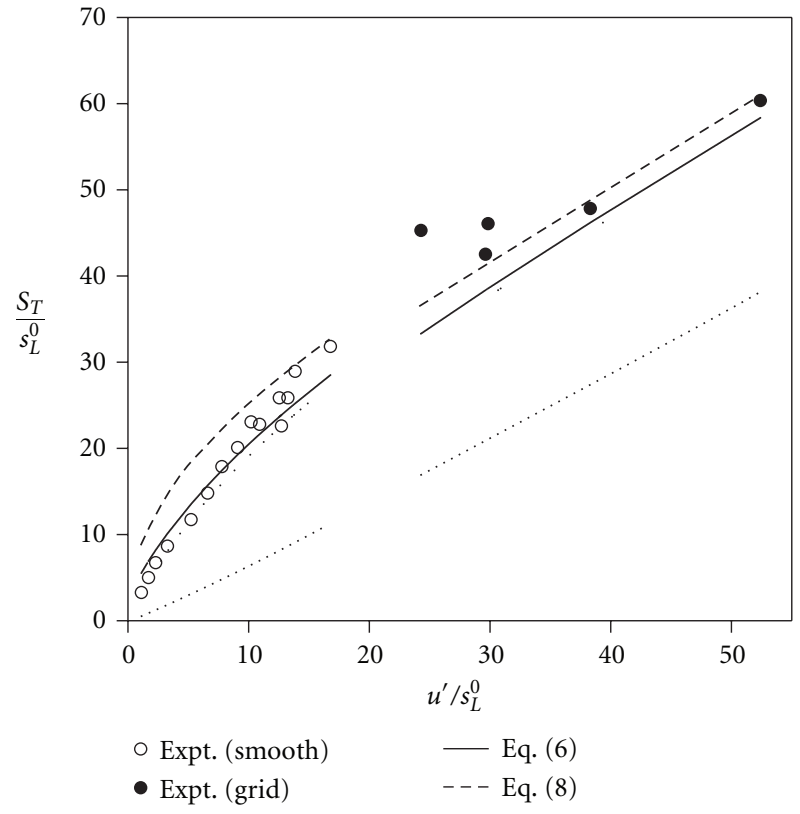

FIGURE 4: Comparisons of turbulent flame speed predictions to the experimental data of $[34,35]$. The open circles denote the part of experiment where an open channel was used and closed circles denote the part where turbulence was generated by a grid. The short dashed line shows comparisons of (8) with $K_{c}^{*}=0$.

completely will give the classical result analysed in many earlier studies.

Comparisons of (6) and (8) with the above three data sets are shown in Figures 2, 3 and 4, respectively. The values of $s_{L}^{o}, \delta_{L}^{o}$, and $\tau$ required for the comparisons are calculated for each case in the same fashion as described in [11]. In general, the predictions of (8) are worse compared to the predictions of (6), and this is expected, since (8) does not include all the effects of density change across the flame front. The quantitative difference in predictions is more pronounced in Figure 2 compared to the other cases. This is because all the data in Figure 2 correspond to low $u^{\prime} / s_{L}^{o}$ values $(\leq 4)$. However, the trend in Figure 3 clearly illustrates the difference between the two expressions. The quantitative difference between (6) and (8) varies from $66 \%$ at $u^{\prime} / s_{L}^{o}=0.3$ in Figure 3(a) to $12 \%$ at $u^{\prime} / s_{L}^{o}=10$ in Figure 3(d). This is further evident in Figure 4, where the quantitative difference decreases from $62 \%$ to $4 \%$ as $u^{\prime} / s_{L}^{o}$ varies from 1.1 to 52.4 . These trends are consistent with the analysis in the previous section, which suggested that neglecting the $C_{4}$ term in (6) affects the scaling appreciably in the low $u^{\prime} / s_{L}^{o}$ limit, while it is negligible in the large $u^{\prime} / s_{L}^{o}$ limit. This observation underscores the effects of density change on the flame speed and the importance of validating turbulent flame speed models with data over a wide range of conditions. If one were to consider experimental data selectively, for instance, data at only large $u^{\prime} / s_{L}^{o}$ in the present case, then the comparisons can be misleading. Furthermore, the dominant effect of gas expansion or density change across the flame front in weak turbulence limit is consistent with the observation by Peters et al. [36] for the corrugated flamelets regime combustion.
Nevertheless, the direct effect of density change prevail for large $u^{\prime} / s_{L}^{o}$ values relevant for thin reaction zones regime of combustion with $\mathrm{Da}>1$. This is becomes clear when the two dashed lines in Figure 4 are compared. The short dashed line in this figure is for (8) with $K_{c}^{*}=0$, which implies that both the direct and indirect effects of heat release are ignored. However, the indirect effect of dilatation becomes negligible only when $u^{\prime} / s_{L}^{o}$ is larger than 50 and $\mathrm{Da}$ is of order unity. This is also clear from Figure 4 (compare the solid and long dashed lines).

\section{Summary and Conclusions}

The present study illustrates the influence of dilatation effects on the propagation speed of turbulent premixed flames, by assessing their contribution to turbulent flame speed calculation. An algebraic model for turbulent flame speed, (6), proposed and validated in earlier studies [11, 12], is a suitable benchmark, since it incorporates the two-way coupling of the effects of density change across the flame front. The direct effect of density change on the local average mixing rate of hot products and cold reactants is through the term involving $K_{c}^{*}$ in (6), and the indirect influence is through the alignment of reactive scalar gradient with the principal components of turbulent strain tensor. The term involving $C_{4}$ in (6) signify the indirect influence.

If both of these terms are ignored, then the original model proposed in $[11,12]$ yields a classical form $S_{T} / s_{L}^{o}=$ $1+C\left(u^{\prime} / s_{L}^{o}\right)$, when the molecular viscosity is included in the analysis, that has been proposed by many early theories. Neglecting only the $K_{c}^{*}$ term but retaining the $C_{4}$ term is unphysical, since it implies the influence of indirect effect when the density change across the flame front is not allowed. Furthermore, this situation leads to negative values for the scalar dissipation rate, $\tilde{\epsilon}_{c}$, violating its realisability condition [12]. On the other hand retaining $K_{c}^{*}$ but neglecting $C_{4}$ yields a flame speed model as in (8), which has a different limiting behaviour compared to the model in (6). The scaling of (8) is different from (6) in the small $u^{\prime} / s_{L}^{o}$ limit but not in the large $u^{\prime} / s_{L}^{o}$ limit. Such a behaviour is also evident in comparisons with experimental data.

The turbulent flame speed values obtained using these two equations differ largely in the limit of weak turbulence (about $66 \%$ for $u^{\prime} / s_{L}^{o} \approx 0.3$ ) or in the corrugated flamelets regime. This is consistent with the observations of Peters et al. [36]. However, the direct effect of dilatation, signified through $K_{c}^{*}$, prevails for large $u^{\prime} / s_{L}^{o}$ values, but the indirect influence of dilatation thorough the dynamics of turbulencescalar interaction weakens as $u^{\prime} / s_{L}^{o}$ increases. These two effects of heat release is observed to prevail for practically relevant values of $u^{\prime} / s_{L}^{o}(\sim 20)$.

\section{References}

[1] A. N. Lipatnikov and J. Chomiak, "Turbulent flame speed and thickness: phenomenology, evaluation, and application in multi-dimensional simulations," Progress in Energy and Combustion Science, vol. 28, no. 1, pp. 1-74, 2002. 
[2] B. Hakberg and A. D. Gosman, "Analytical determination of turbulent flame speed from combustion models," Proceedings of the Combustion Institute, vol. 20, pp. 225-232, 1984.

[3] C. A. Catlin and R. P. Lindstedt, "Premixed turbulent burning velocities derived from mixing controlled reaction models with cold front quenching," Combustion and Flame, vol. 85, no. 3-4, pp. 427-439, 1991.

[4] J. M. Duclos, D. Veynante, and T. J. Poinsot, "A comparison of flamelet models for premixed turbulent combustion," Combustion and Flame, vol. 95, no. 1-2, pp. 101-117, 1993.

[5] N. Peters, "The turbulent burning velocity for large-scale and small-scale turbulence," The Journal of Fluid Mechanics, vol. 384, pp. 107-132, 1999.

[6] V. L. Zimont, "Gas premixed combustion at high turbulence. Turbulent flame closure combustion model," Experimental Thermal and Fluid Science, vol. 21, no. 1-3, pp. 179-186, 2000.

[7] Ö. L. Gülder, "Turbulent premixed flame propagation models for different combustion regimes," Proceedings of the Combustion Institute, vol. 23, pp. 743-750, 1990.

[8] K. N. C. Bray, "Studies of the turbulent burning velocity," Proceedings of the Royal Society A, vol. 431, no. 1882, pp. 315$335,1990$.

[9] K. N. C. Bray, "Turbulent flows with premixed reactants," in Turbulent Reacting Flows, P. A. Libby and F. A. Williams, Eds., pp. 115-183, Springer, New York, NY, USA, 1980.

[10] R. W. Bilger, M. B. Esler, and S. H. Starner, "On reduced mechanisms for methane-air combustion," in Reduced Kinetic Mechanisms and Asymptotic Approximations for Methane-Air Flames, M. D. Smooke, Ed., p. 86, Springer, Berlin, Germany, 1991.

[11] H. Kolla, J. W. Rogerson, and N. Swaminathan, "Validation of a turbulent flame speed model across combustion regimes," Combustion Science and Technology, vol. 182, no. 3, pp. 284308,2010 .

[12] H. Kolla, J. W. Rogerson, N. Chakraborty, and N. Swaminathan, "Scalar dissipation rate modeling and its validation," Combustion Science and Technology, vol. 181, no. 3, pp. 518$535,2009$.

[13] Y. B. Zeldovich, G. I. Barenblatt, V. B. Librovich, and G. M. Makhviladze, The Mathematical Theory of Combustion and Explosions, Consultants Bureau, New York, NY, USA, 1985, translated from Russian by D. H. McNeill.

[14] C. Corvellec, P. Bruel, and V. A. Sabel'nikov, "Turbulent premixed flames in the flamelet regime: burning velocity spectral properties in the presence of countergradient diffusion," Combustion and Flame, vol. 120, no. 4, pp. 585-588, 2000.

[15] N. Swaminathan and K. N. C. Bray, "Effect of dilatation on scalar dissipation in turbulent premixed flames," Combustion and Flame, vol. 143, no. 4, pp. 549-565, 2005.

[16] N. Swaminathan and R. W. Grout, "Interaction of turbulence and scalar fields in premixed flames," Physics of Fluids, vol. 18, no. 4, Article ID 045102, pp. 1-9, 2006.

[17] N. Chakraborty and N. Swaminathan, "Influence of the Damköhler number on turbulence-scalar interaction in premixed flames. I. Physical insight," Physics of Fluids, vol. 19, no. 4, Article ID 045103, 2007.

[18] H. S. Kim and H. Pitsch, "Scalar gradient and small-scale structure in turbulent premixed combustion," Physics of Fluids, vol. 19, no. 11, Article ID 115104, 14 pages, 2007.

[19] A. Mura and M. Champion, "Relevance of the Bray number in the small-scale modeling of turbulent premixed flames," Combustion and Flame, vol. 156, no. 3, pp. 729-733, 2009.

[20] A. Mura, V. Robin, M. Champion, and T. Hasegawa, "Small scale features of velocity and scalar fields in turbulent premixed flames," Flow, Turbulence and Combustion, vol. 82, no. 3, pp. 339-358, 2009.

[21] A. N. Lipatnikov and J. Chomiak, "Effects of premixed flames on turbulence and turbulent scalar transport," Progress in Energy and Combustion Science, vol. 36, no. 1, pp. 1-102, 2010.

[22] N. Chakraborty, J. W. Rogerson, and N. Swaminathan, "A priori assessment of closures for scalar dissipation rate transport in turbulent premixed flames using direct numerical simulation," Physics of Fluids, vol. 20, no. 4, Article ID 045106, 2008.

[23] N. Chakraborty, M. Klein, and N. Swaminathan, "Effects of Lewis number on the reactive scalar gradient alignment with local strain rate in turbulent premixed flames," Proceedings of the Combustion Institute, vol. 32, no. 1, pp. 1409-1417, 2009.

[24] N. Chakraborty and N. Swaminathan, "Influence of the Damköhler number on turbulence-scalar interaction in premixed flames. II. Model development," Physics of Fluids, vol. 19, no. 4, Article ID 045104, 11 pages, 2007.

[25] N. Chakraborty, J. W. Rogerson, and N. Swaminathan, "The scalar gradient alignment statistics of flame kernels and its modelling implications for turbulent premixed combustion," Flow, Turbulence and Combustion, vol. 85, no. 1, pp. 25-55, 2010.

[26] E. S. Richardson, R. Sankaran, R. W. Grout, and J. H. Chen, "Numerical analysis of reaction-diffusion effects on species mixing rates in turbulent premixed methane-air combustion," Combustion and Flame, vol. 157, no. 3, pp. 506-515, 2010.

[27] G. Hartung, J. Hult, C. F. Kaminski, J. W. Rogerson, and N. Swaminathan, "Effect of heat release in turbulence and scalarturbulence interaction in premixed combustion," Physics of Fluids, vol. 20, no. 9, Article ID 035110, 2008.

[28] P. A. Libby, "Characteristics of laminar lifted flames in a partially premixed jet," Combustion Science and Technology, vol. 68, no. 1-3, pp. 15-33, 1989.

[29] G. Damköhler, "Der Einfluss der Turbulenz auf die Flammengeschwindigkeit in Gasgemischen," Zeitschrift für Elektrochemie und Angewandte Physikalische Chemie, vol. 46, no. 11, pp. 601-626, 1940, (English Translation NASA TM 1112, 1947).

[30] P. Clavin and F. A. Williams, "Effects of molecular diffusion and of thermal expansion on the structure and dynamics of premixed flames in turbulent flows of large scale and low intensity," The Journal of Fluid Mechanics, vol. 116, pp. 251282, 1982.

[31] R. C. Aldredge, V. Vaezi, and P. D. Ronney, "Premixed-flame propagation in turbulent Taylor-Couette flow," Combustion and Flame, vol. 115, no. 3, pp. 395-405, 1998.

[32] J. F. Driscoll, "Turbulent premixed combustion: flamelet structure and its effect on turbulent burning velocities," Progress in Energy and Combustion Science, vol. 34, no. 1, pp. 91-134, 2008.

[33] H. Kobayashi, T. Tamura, K. Maruta, T. Niioka, and F. A. Williams, "Burning velocity of turbulent premixed flames in a high-pressure environment," Proceedings of the Combustion Institute, vol. 26, pp. 389-396, 1996.

[34] S. M. Il'yashenko and A. V. Talantov, "Theory and analysis of straight-through-flow combustion chambers," Tech. Rep. TT6662119, Wright Patterson AFB, Dayton, Ohio, USA, 1966.

[35] S. R. Turns, An Introduction to Combustion, Concepts and Applications, McGraw-Hill, Singapore, 2nd edition, 2000.

[36] N. Peters, H. Wenzel, and F. A. Williams, "Modification of the turbulent burning velocity by gas expansion," Proceedings of the Combustion Institute, vol. 18, Article ID 045102, 9 pages, 2006. 

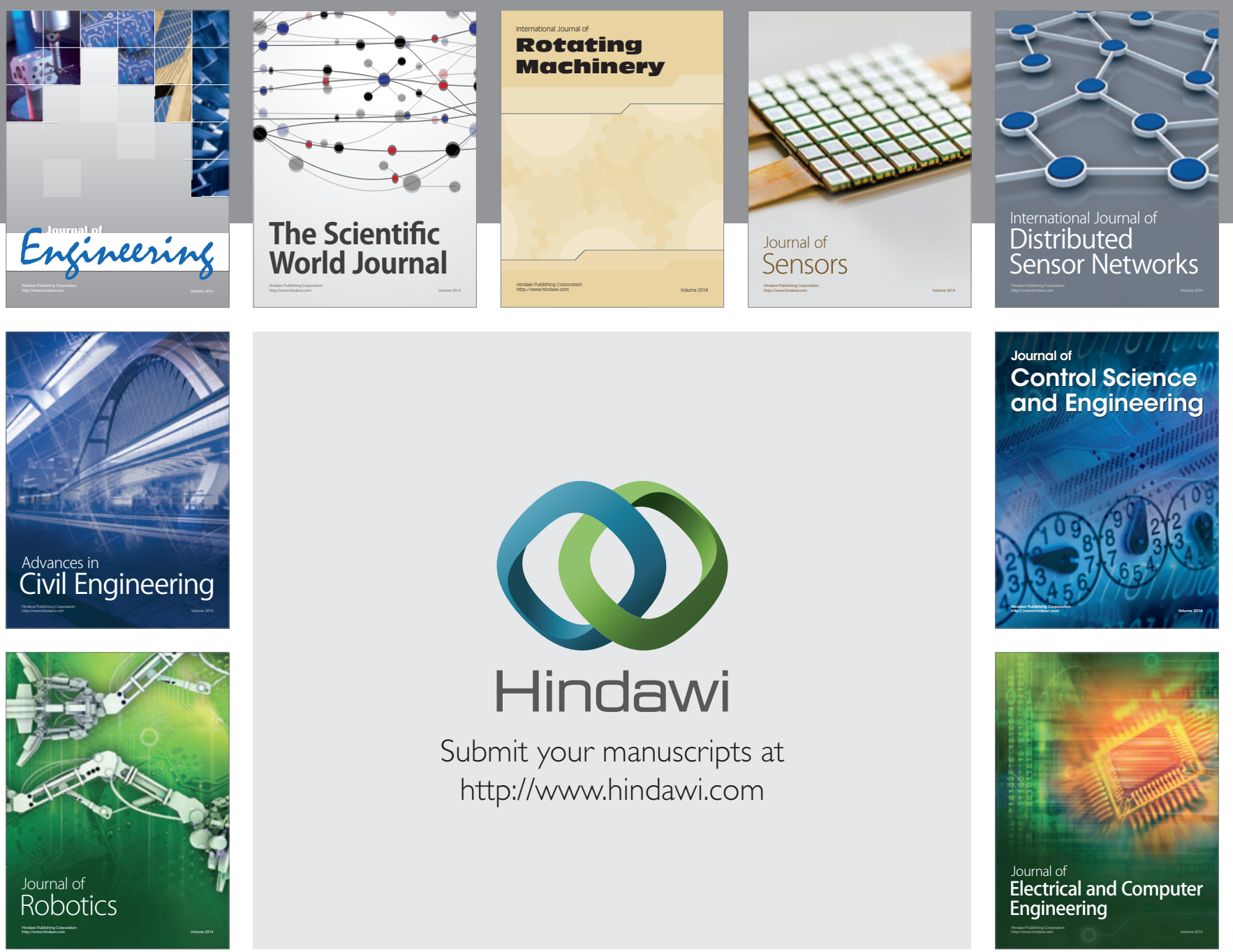

Submit your manuscripts at

http://www.hindawi.com
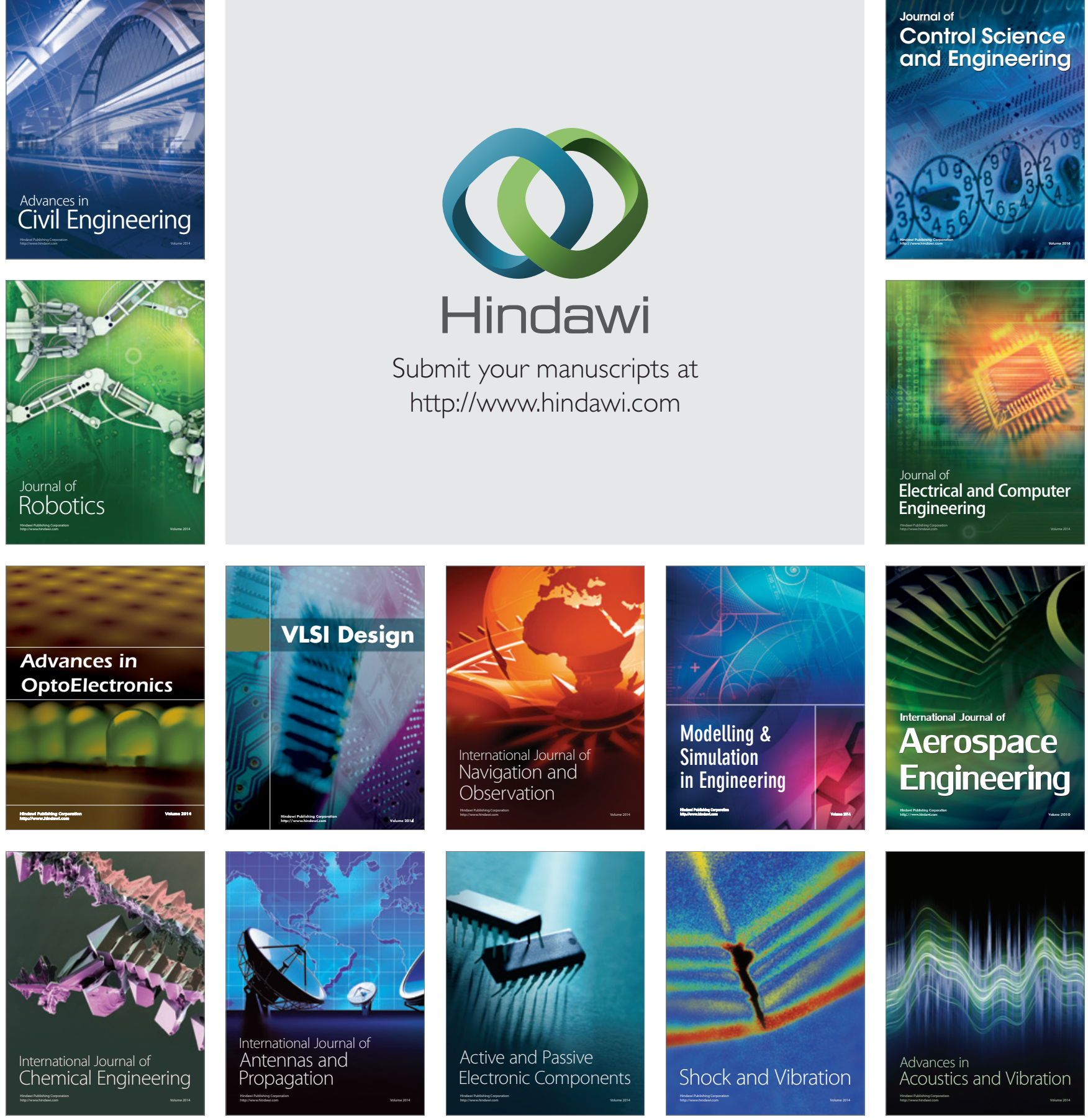